\title{
Characterization and evaluation of microsatellite markers in a strain of the oriental fruit fly, Bactrocera dorsalis (Diptera: Tephritidae), with a genetic sexing character used in sterile insect population control
}

\author{
Siriwan ISASAWIN, Nidchaya AKETARAWONG and SuJinda THANAPHUM* \\ Department of Biotechnology, Faculty of Science, Mahidol University, Rama VI Road, Bangkok 10400, Thailand
}

Key words. Tephritidae, oriental fruit fly, Bactrocera dorsalis, microsatellites, genetic sexing strain, mating competitiveness, sterile insect technique

\begin{abstract}
The oriental fruit fly, Bactrocera dorsalis, is a key economic insect pest reducing fruit yield and generating constraints in the international market. The application of the sterile insect technique (SIT) continues to reveal areas where new technologies can improve the effectiveness of fruit fly control. One such advancement concerns insect strains. In the present study, a mass-reared strain of the fly with a translocation-based genetic sexing character (Salaya1) based on a brown-white pupal colour dimorphism was genetically characterized using 11 microsatellite DNA markers. Subsequently, these markers were used to evaluate the maintenance of genetic variability in the strain under mass-rearing conditions. Mating competitiveness of this strain was also tested in field cages. Two of the newly characterized Y-pseudo-linked microsatellite markers were used for strain identification in field monitoring traps. The strain was also validated in a pilot integrated pest management (IPM) programme using male-only SIT in a fruit orchard. The programme resulted in the suppression of the fruit fly population.
\end{abstract}

\section{INTRODUCTION}

The oriental fruit fly, Bactrocera dorsalis (Hendel) (Diptera: Tephritidae), is a key economic pest causing reductions of fruit yields and generating constraints in the international market (Clarke et al., 2005). Its geographical distribution is expanding in tropical and subtropical regions from Asia to a potentially much larger range (CABI/EPPO, 1998). The oriental fruit fly is invasive due to its apparent wide host range, high fecundity, dispersal capacity, and bioclimatic adaptive abilities (Aketarawong et al., 2007; Stephens et al., 2007). Integrated pest management (IPM) of the oriental fruit fly coordinates the use of pest and environmental information and available pest control methods to prevent economic losses whilst hopefully reducing negative effects to the environment.

General fruit fly control methods include application of insecticide spray, male annihilation traps (MAT), wild host cutting, fruit wrapping, and fruit orchard phytosanitation practices. Although most of these control measures are used in combination and are practical for immediate pest suppression, they impose either an environmental impact, health hazards, and/or are labour intensive. A highly effective birth control technology such as sterile insect technique (SIT) is usually incorporated in order to increase the impact of pest management and reduce insecticide problems (Klassen, 2005). There are many successful examples of pest control programmes using SIT worldwide (Klassen \& Curtis, 2005). In relation to the oriental fruit fly, the SIT method was first integrated in area-wide control programmes about ten years ago (Orankanok et al., 2007).
SIT involves a mass release of sterile insects in an attempt to win the overall mating competition among fertile insects in the field. This is a birth control practice that reduces the mating between fertile flies in the target area and thus reduces the size of the next generation. Unlike chemical control methods, SIT is target-species specific. It has no off-target effects on the ecosystem or human health. This makes the approach compatible with organic farming (Wimmer, 2005). In addition, the sterile flies are mobile control agents that seek mates even in areas that cannot be reached by insecticides and beyond the immediate area of application (Hendrichs et al., 2007).

Operational use of SIT continues to reveal areas where new technologies are needed to improve efficiency and thus lead to more cost effective programmes. One critical area identified by SIT exponents where major advancement could be made concerns genetic improvement of insect strains. For any effective SIT programme, established strains are required as parental colonies. They should be massively produced but able to retain vigour when released throughout the field. Their mating competitiveness should also be as effective as that of the wild flies. It is also desirable for purposes of monitoring to have an intrinsic identification system for identification of sterile flies when they are recaptured together with wild flies. An example of strain improvement that has significant global impact on SIT programmes is the application of genetic sexing strains (GSS) against the Mediterranean fruit fly in area-wide integrated pest management (AWIPM) programmes (Robinson, 2002). Removal of females from the release material increases the efficiency of the

\footnotetext{
* Corresponding author; e-mail: testn@mahidol.ac.th
} 
application because mating among released insects is eliminated, since only males are then released (Franz, 2005). Furthermore, SIT is more acceptable to the end users as releasing males only reduces stinging of fruits compared to bisexual releases.

An additional improvement is the development of genetic markers that can be used for strain identification. These markers allow discrimination of released from wild flies in monitoring traps and/or to identify the presence of sterile male sperms in the spermathecae of wild females (San Andrés et al., 2007; Scolari et al., 2008). Furthermore, the genetic markers can be employed as analytical tools for strain colonization and quality management. More especially because the loss of genetic diversity during mass rearing may result in the loss of vigour and mating compatibility with wild flies.

Microsatellite markers are repetitive DNA sequences of $2-6$ base pairs in length. They are usually found in noncoding DNA sequences and are widely distributed and abundantly found on the eukaryotic chromosome (Goldstein \& Schlötterer, 1999; Loxdale \& Lushai, 1998). They are suitable and sensitive tools for studying recent population changes because they are essentially selectively neutral and often highly polymorphic (Guichoux et al., 2011). Monitoring in ecological pest management, such as identification of individual flies, is often performed using such markers (Waits et al., 2001). In recent years, high resolution microsatellite markers for $B$. dorsalis were designed (Aketarawong et al., 2006). Many of these markers revealed high levels of polymorphism in different geographical populations, either at micro- or macro- geographical levels (Aketarawong et al., 2007). Subsequently, they were utilized for the evaluation of AW-IPM using SIT for fruit fly control programmes (Aketarawong et al., 2011).

In the present study, we analyzed microsatellite markers of a strain which is a translocation-based genetic sexing strain (Salaya1) based on a brown-white pupal color dimorphism. Subsequently, suitable markers were used to evaluate the maintenance of genetic variability of this strain under mass-rearing conditions. The mating competiveness of this strain was also compared to wild fruit flies in field cage experiments. Two of the newly characterized Y-pseudo-linked microsatellite markers were utilized for strain identification in field-monitoring traps. The effectiveness of the strain was then validated in a pilotscale IPM programme using male-only SIT.

\section{MATERIAL AND METHODS}

\section{Mass-reared Salaya1 strain}

Salayal is a Y-autosome translocation-based genetic sexing strain of the oriental fruit fly based on a brown-white pupal color dimorphism. This strain was developed following the McCombs \& Saul (1995) screening method for B. dorsalis. The males and the females can be sorted by their brown- and whitepupal colors, respectively.

A genetic sexing strain contains two principle components, a selectable marker (e.g. white pupae) and a reciprocal Y-autosome translocation linking the autosome carrying the wild type allele of the selectable marker to the male determining

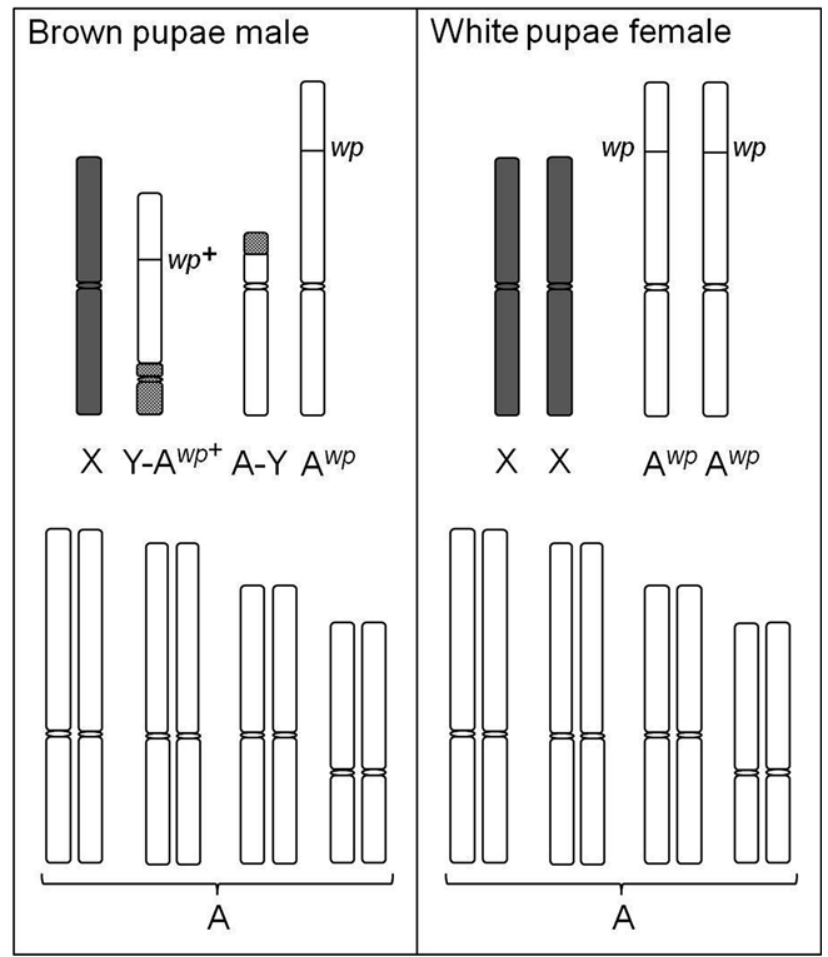

Fig. 1. Schematic representation of the entire chromosomal complement for a basic Y-autosome translocation based genetic sexing strain. $w p^{+}$represents the wild type while $w p$ refers to the mutant allele. $\mathrm{A}^{w p}$ represents the free autosome carrying the mutant allele. $\mathrm{Y}-\mathrm{A}^{w p^{+}}$and $\mathrm{A}-\mathrm{Y}$ represent the two reciprocal components of the $\mathrm{Y}$-autosome translocation. The location of the $\mathrm{Y}$ chromosomal and the autosomal centromere of the translocation are not known for Salaya1.

Y chromosome. GSS males are heterozygous for the selectable marker, i.e. the free autosome carries the mutant allele and the translocated homologue the wild type allele. The GSS females are homozygous for the marker (Fig. 1). Genetic instability of GSS is the result of pre-meiotic recombination in the heterozygous, translocation carrying males. Type-1 recombination (recombination in the chromosomal region between the translocation breakpoint and the selectable marker) results in two reciprocal recombinant types, females emerging from brown and males emerging from white pupae (Franz, 2005). Therefore, the genetic stability of the GSS can be checked every generation by inspecting 100 brown and white pupae as well as the sex of the flies emerging from these pupae.

White pupae involves an autosomal recessive mutation and was detected by screening large numbers of flies of the mass reared strain. The homozygous white pupae strain was established with very few individuals. The $\mathrm{Y}$ autosome translocation was induced by irradiating wild type males with cobalt-60, 10 Gy. The treated males were mass mated with white pupae females followed by 100 single male crosses between the $F_{1}$ males and white pupae females. The progeny was screened for families with pseudo-linkage between white pupae and sex. One family was detected.

\section{Mass-rearing conditions}

The Salayal strain was reared in a mass-rearing cage with the dimensions of $0.4 \times 1.0 \times 1.8 \mathrm{~m}$ (width $\times$ length $\times$ height) at $25^{\circ} \mathrm{C}$ and $13 \mathrm{~L}: 11 \mathrm{D}$ cycles. Approximately 4,000 males and 13,000 females produced 1.5 million eggs monthly and yielded some 150,000 adults. The use of higher proportion of females 
was chosen to maximize egg yield in the mass-rearing process. The adult food consisted of $75 \%(\mathrm{w} / \mathrm{w})$ sucrose and $25 \%(\mathrm{w} / \mathrm{w})$ yeast extract. Each batch of approximately 75,000 eggs was overlaid on the top of a larval food tray with the dimensions of $0.3 \times 0.2 \times 0.025 \mathrm{~m}$ (width $\times$ length $\times$ height $).$ The larval food consisted of $29.5 \%(\mathrm{w} / \mathrm{w})$ wheat bran, $13.8 \%(\mathrm{w} / \mathrm{w})$ sucrose $4.0 \%(\mathrm{w} / \mathrm{w})$ yeast extract, $0.2 \%(\mathrm{w} / \mathrm{w})$ sodium benzoate, and $52.5 \%(\mathrm{w} / \mathrm{w})$ water. For SIT experiments, pupae were irradiated with 50 Gy during their late pupal stage. The newly emerged adults were fed with adult food for 2-3 days before their release. No treatment to increase mating competitiveness was applied.

\section{Fruit fly sample collection and genomic DNA extraction}

Thirty individuals of each sex were collected in generations $F_{5}$ and $F_{17}$ from the mass-reared Salayal colony for the genetic variation analyses using polymorphic microsatellites. Also, wild males were trapped using Steiner traps (IAEA, 2003) or wild flies were collected from infested mangoes from an experimental orchard in Nakhon Pathom Province, Thailand, during 2009. The mixture of $75 \%(\mathrm{v} / \mathrm{v})$ methyl eugenol (ME) and $25 \%$ $(\mathrm{v} / \mathrm{v})$ insecticide, cypermethrin, was applied as a male lure. Thirty-five wild males were used for genetic analyses. The samples were preserved in $95 \%$ ethanol and stored at $-20^{\circ} \mathrm{C}$ before DNA extraction. Genomic DNA was extracted from individual flies as detailed in Aketarawong et al. (2007).

\section{Microsatellite analysis}

Amplifications of six B. dorsalis microsatellite loci; Bd1, $\mathrm{Bd} 9, \mathrm{Bd} 15, \mathrm{Bd} 19, \mathrm{Bd} 42$, and $\mathrm{Bd} 85 \mathrm{~B}$ (Aketarawong et al., 2006); and five B. papayae loci; Bp58, Bp73, Bp125, Bp173, and Bp181 (Shearman et al., 2006) were performed with each DNA sample by polymerase chain reaction (PCR) according to the PCR conditions as described in Aketarawong et al. (2006). PCR products were resolved on $6 \%$ or $12 \%$ polyacrylamide electrophoresis gels, depending on microsatellite locus size. Allele sizes were determined by comparison with a 25 base pairs ladder (Promega, Madison, USA).

\section{Statistical analysis of genetic variation}

The genetic variability of each population sample was examined in terms of the average number of alleles $\left(N_{\mathrm{a}}\right)$, as well as average observed and expected heterozygosity $\left(H_{\mathrm{O}}\right.$ and $\left.H_{\mathrm{E}}\right)$. These parameters were estimated using Microsatellite Analyser (MSA) software v. 4.05 (Dieringer \& Schlötterer, 2003).

Hardy-Weinberg equilibrium deviations were evaluated in the mass-reared populations after Bonferroni correction (Rice, 1989) $(P<0.05)$ using GENEPOP software v. 4.0 (Rousset, 2008).

Allele frequencies from generations $F_{5}$ and $F_{17}$ of the Salaya1 strain were compared using contingency table chi-square tests $(\alpha=0.05)$. The average observed heterozygosity ( \pm standard error, SE) was calculated from bootstrapping (10 replicates). A $t$-test was used to compare these average observed heterozygosity levels using PASW statistics software v. 18.0 (or SPSS; 2009, Chicago, IL, USA; http://www.spss.com).

\section{Field cage test setting}

Field cages with the standard dimensions of $3.0 \times 3.5 \times 2.3 \mathrm{~m}$ (width $\times$ length $\times$ height) were set up with a mango tree inside. According to standard protocol "Required periodic quality control tests" from FAO/IAEA/USDA (2003), wild flies were either directly collected from the field or from a colony that had recently descended from wild flies. In order to determine if the tested flies were sexually mature prior to the field cage releasing, the approximately 12 day-old flies were checked to see if they displayed courtship behaviour in the laboratory. Sexually mature flies were released into field cages with food
TABle 1. Type, gender, and number of fruit flies in field cages.

\begin{tabular}{lccccc}
\hline & \multicolumn{4}{c}{ Released fruit fly types } \\
\cline { 2 - 3 } Treatments & \multicolumn{2}{c}{ Wild flies } & & Mass-reared strain \\
\cline { 2 - 3 } \cline { 5 - 6 } & $\begin{array}{c}\text { Fertile } \\
\text { males }\end{array}$ & $\begin{array}{c}\text { Fertile } \\
\text { females }\end{array}$ & & $\begin{array}{c}\text { Sterile } \\
\text { males }\end{array}$ & $\begin{array}{c}\text { Sterile } \\
\text { females }\end{array}$ \\
\hline I. Baseline control & 20 & 20 & - & - \\
II. Bisexual SIT & 20 & 20 & & 20 & 20 \\
III. Male-only SIT & 20 & 20 & & 20 & - \\
IV. Baseline MAT & 5 & 20 & & - \\
V. MAT + Bisexual SIT & 5 & 20 & & 15 & 15 \\
VI. MAT + Male-only SIT & 5 & 20 & & 15 & - \\
\hline
\end{tabular}

and water. Four egg ball devices were hung on the mango tree. The devices were globular in shape, approximately $3.5 \mathrm{~cm}$. in diameter. They were made of $0.7 \%$ (w/v) agar powder with $25 \%$ $(\mathrm{v} / \mathrm{v})$ mango juice and $75 \%(\mathrm{v} / \mathrm{v})$ water. Wild and sterile males were firstly released into the cages and given 15-30 min to disperse and establish territories. The flies were released before dusk (approximately 5:45 to 6:15 p.m.), the time of peak mating. The tests were run for at least two days. At $48 \mathrm{~h}$ after placing the flies into the field cages, infested egg ball devices were replaced. Eggs were placed onto paper on top of the larval food cup. The eggs were incubated at $25^{\circ} \mathrm{C}$ and the egg hatch observed after two days.

\section{Experimental design and statistical analysis for field cage testing}

The objective of the experiment was to test if the mating competition of sterile males versus the fertile wild males can generate enough sterility of the wild females under natural mating conditions. Two sets of experiments were conducted, without or with simulated male annihilation traps (MAT) (treatments 1-3 and 4-6, respectively; Table 1). Each set consisted of the release of only fertile flies, adding sterile males and females, and adding only sterile males in the release. The baseline sterility level was taken from the baseline control treatment. The second treatment was to test the release of a sterile bisexual strain, whereas the third treatment was to test the release of only sterile males.

The MAT scenario was tested as the fourth treatment. A reduction of fertile wild males from 20 to 5 individuals was arbitrarily set up in this set of experiments. The combination of MAT and bisexual SIT practices was tested as the fifth treatment. Likewise, the combination of the MAT and male-only SIT practice was tested as the sixth treatment. These treatments also arbitrarily reduced the number of sterile flies from 20 to 15 flies because the number of sterile flies could be reduced by MAT in real situation (Table 1).

Three replications were tested for each treatment. A $t$-test was used to compare the average percentage of egg hatch $( \pm \mathrm{SE})$ among the six treatments using PASW statistics software v. 18.0 .

\section{IPM using SIT in the pilot field test}

Traps were set up at high density (every $50 \mathrm{~m}$ ) for monitoring. The dimensions of the fruit orchard chosen was approximately $250 \times 100 \mathrm{~m}$. Weekly fruit fly monitoring was performed for a year to record baseline data. Fruit damage was determined throughout the fruit orchard following the Orankanok et al. (2007) monitoring method. Two months before the male-only SIT application was started, fruit orchard phytosanitation practices such as MAT, picking up fruit under the fruit 
TABLE 2. Genotypic frequencies of microsatellite loci observed in the Salaya1 strain and a wild male population.

\begin{tabular}{|c|c|c|c|c|}
\hline \multirow{2}{*}{ Locus } & \multirow{2}{*}{ Genotypes } & \multicolumn{3}{|c|}{ Frequency } \\
\hline & & Salayal males $\mathrm{F}_{5}$ & Salayal females $F_{5}$ & Wild males \\
\hline \multirow[t]{4}{*}{$\mathrm{Bd} 9$} & $169 / 169$ & 0.73 & 0.67 & 0.09 \\
\hline & $169 / 170$ & 0.17 & 0.30 & 0.06 \\
\hline & $170 / 170$ & 0.10 & 0.03 & 0.06 \\
\hline & all other genotypes & none & none & 0.79 \\
\hline \multirow[t]{7}{*}{ Bd19 } & $157 / 157$ & 0.00 & 0.07 & 0.00 \\
\hline & $157 / 162$ & 0.07 & 0.03 & 0.00 \\
\hline & $157 / 168$ & 0.03 & 0.07 & 0.00 \\
\hline & $162 / 162$ & 0.27 & 0.40 & 0.00 \\
\hline & $162 / 168$ & 0.53 & 0.23 & 0.00 \\
\hline & $168 / 168$ & 0.10 & 0.20 & 0.03 \\
\hline & all other genotypes & none & none & 0.97 \\
\hline \multirow[t]{3}{*}{$\mathrm{Bd} 85 \mathrm{~B}$} & $85 / 85$ & 0.90 & 0.87 & 0.11 \\
\hline & $85 / 89$ & 0.10 & 0.13 & 0.00 \\
\hline & all other genotypes & none & none & 0.89 \\
\hline \multirow[t]{4}{*}{ Bp173 } & $131 / 131$ & 0.10 & 0.03 & 0.00 \\
\hline & $131 / 135$ & 0.47 & 0.50 & 0.00 \\
\hline & $135 / 135$ & 0.43 & 0.47 & 0.03 \\
\hline & all other genotypes & none & none & 0.97 \\
\hline \multirow[t]{2}{*}{$\overline{\mathrm{Bd} 1}$} & $105 / 105$ & 1.00 & 1.00 & 0.03 \\
\hline & all other genotypes & none & none & 0.97 \\
\hline \multirow[t]{2}{*}{ Bp125 } & $103 / 103$ & 1.00 & 1.00 & 0.11 \\
\hline & all other genotypes & none & none & 0.89 \\
\hline \multirow[t]{2}{*}{ Bp181 } & $126 / 126$ & 1.00 & 1.00 & 0.14 \\
\hline & all other genotypes & none & none & 0.86 \\
\hline \multirow[t]{3}{*}{$\mathrm{Bd} 15$} & $191 / 191$ & 0.00 & 1.00 & 0.00 \\
\hline & $191 / 192$ & 1.00 & 0.00 & 0.00 \\
\hline & all other genotypes & none & none & 1.00 \\
\hline \multirow[t]{4}{*}{$\mathrm{Bd} 42$} & $181 / 181$ & 0.00 & 0.27 & 0.26 \\
\hline & $181 / 186$ & 0.47 & 0.60 & 0.00 \\
\hline & $186 / 186$ & 0.53 & 0.13 & 0.00 \\
\hline & all other genotypes & none & none & 0.74 \\
\hline \multirow[t]{3}{*}{$\mathrm{Bp} 58$} & $116 / 116$ & 0.00 & 1.00 & 0.11 \\
\hline & $116 / 118$ & 1.00 & 0.00 & 0.00 \\
\hline & all other genotypes & none & none & 0.89 \\
\hline \multirow[t]{3}{*}{$\mathrm{Bp} 73$} & $111 / 111$ & 0.00 & 1.00 & 0.09 \\
\hline & $111 / 113$ & 1.00 & 0.00 & 0.09 \\
\hline & all other genotypes & none & none & 0.82 \\
\hline
\end{tabular}

trees, and wild host cutting were applied. These practices were undertaken to reduce the fruit fly number before the application of the SIT. The phytosanitation practices were also regularly carried out while approximately 40,000 sterile male fruit flies were released bimonthly. When fruit fly hot spots were identified, wild host cutting and fruit garbage dumping site searches were done. However, the application of insecticides and fruit wrapping had never been applied to this particular fruit orchard.

\section{Fruit fly monitoring and strain identification}

Fifteen Steiner traps were checked and the ME and insecticide were replaced once a week. The fruit flies were also collected for DNA extraction once a week. Molecular strain identification was done using $\mathrm{Bd} 42$ and $\mathrm{Bp} 73$ microsatellite markers. The average wild fruit flies/trap/day were recorded.

\section{RESULTS}

\section{Characterization of microsatellite DNA markers in the Salaya1 strain}

The overall genetic variation was estimated from $N_{\mathrm{a}}$, $H_{\mathrm{O}}$, and $H_{\mathrm{E}}$, obtained using the 11 microsatellite loci. The wild fruit fly population showed a much higher average number of alleles per locus than the Salayal strain $\left(N_{\mathrm{a}}=\right.$ 11.36 and 1.82 , respectively). Likewise, average observed and expected heterozygosities per locus were higher in the wild population than the Salayal strain $\left(H_{\mathrm{O}}=0.52\right.$ and $0.44 ; H_{\mathrm{E}}=0.83$ and 0.30 , respectively).

Four microsatellite loci (Bd9, Bd19, Bd85B, and Bp173) showed polymorphic patterns and three loci (Bd1, Bp125, and Bp181) appeared to have fixed alleles in the Salayal strain (Table 2). The other four microsatellite loci (Bd15, Bd42, Bp58, and Bp73) appeared to provide a unique genotypic pattern only in the mass-reared Salayal strain. Three of these loci, Bd15, Bp58, and Bp73, showed characteristics of fixed heterozygotes that seemed specific to only male genotypes. These markers are located on the autosome-carrying white pupae mutation. Furthermore, the white pupae strain is apparently homozygous for alleles 111, 116, and 191, while the translocated homologue carries alleles 113, 118, and 192. Bd42 

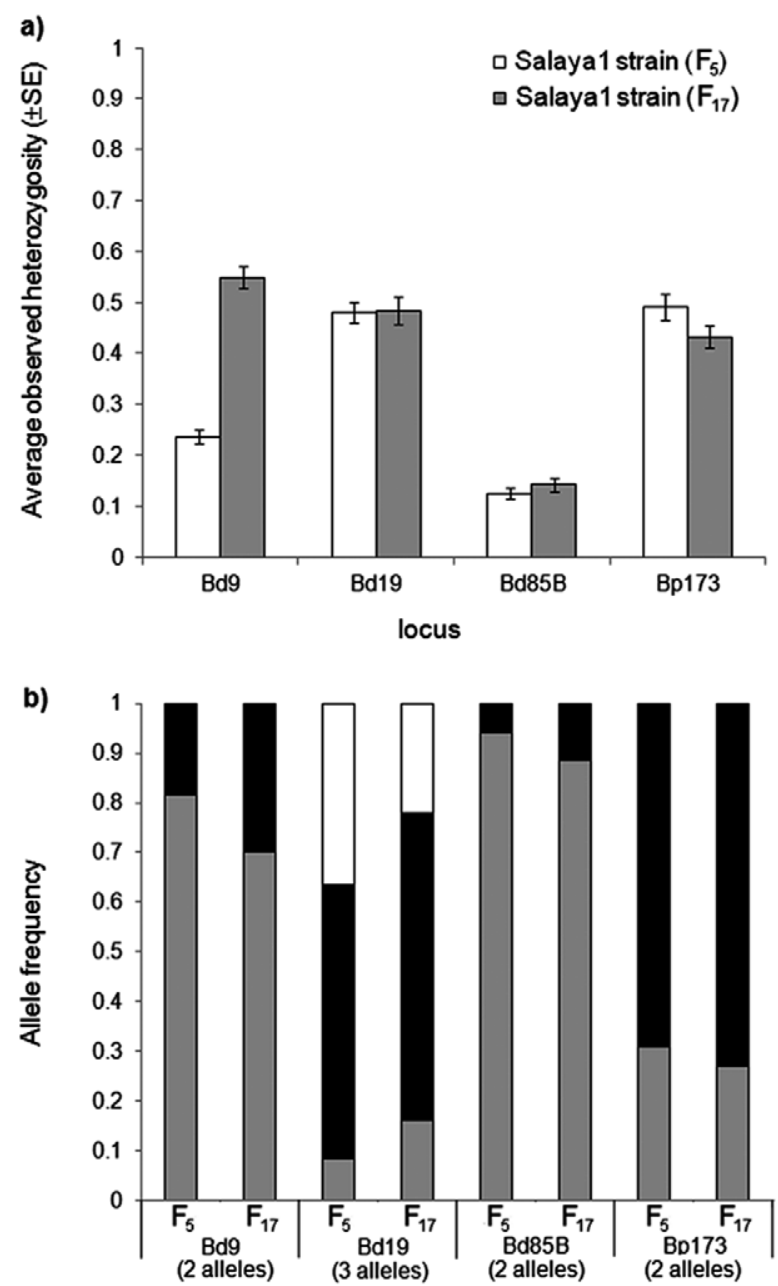

Fig. 2. Comparison of genetic variation level between generations $\mathrm{F}_{5}$ and $\mathrm{F}_{17}$ of the Salayal strain from the mass-rearing facility: a) average observed heterozygosity $( \pm \mathrm{SE})$; b) allele frequency.

is a bit more complicated: it seems that the allele 186 is located in the autosome segment translocated onto the $\mathrm{Y}$ chromosome, while the free autosomes show a mixture of alleles 181 and 186. The possibility of using malespecific genotypes for strain identification in the maleonly sterile insect release was evaluated using data from a wild male population. Although the genotypes 111/113, $116 / 118,191 / 192$, and $181 / 186$ (or 186/186) individuals could theoretically be found in the target wild male population, the observed genotypic frequencies would be very low (Table 2).

\section{Evaluation of the maintenance of the genetic variability of the mass-reared strain}

The average observed heterozygosities of the Bd19, $\mathrm{Bd} 85 \mathrm{~B}$, and $\mathrm{Bp} 173$ loci, between the generations $\mathrm{F}_{5}$ and $\mathrm{F}_{17}$, were not significantly different $(t=-0.09$, d.f. $=18, P$ $=0.933 ; t=-1.05$, d.f. $=18, P=0.307 ; t=1.17$, d.f. $=18$, $P=0.093$, respectively) (Fig. 2a). However, the average observed heterozygosity of the Bd9 locus in generation $\mathrm{F}_{5}$ was significantly lower than the observed heterozygosity in generation $\mathrm{F}_{17},(t=-12.16$, d.f. $=18, P=0.000)$. There was also no significant difference in the frequencies of all

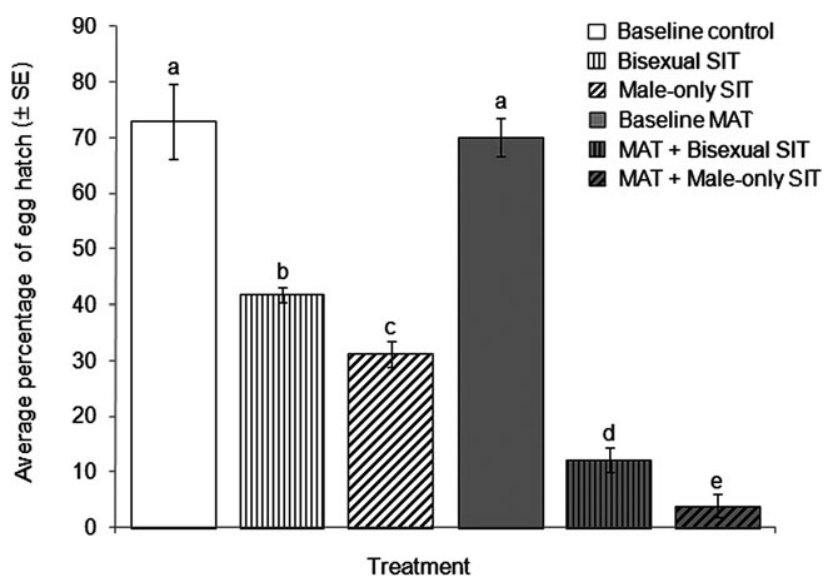

Fig. 3. Average percentage of egg hatch $( \pm$ SE) of wild females from field cages among six treatments: different letters represent significant differences $(\alpha=0.05)$ after $t$-test for equality of means.

alleles at loci $\mathrm{Bd} 9, \mathrm{Bd} 19, \mathrm{Bd} 85 \mathrm{~B}$, and $\mathrm{Bp} 173$ between the two generations $\left(\chi^{2}=3.44<\chi_{0.05[1]}^{2}=3.84 ; \chi^{2}=5.69<\right.$ $\chi^{2}{ }_{0.05[2]}=5.99 ; \chi^{2}=1.90<\chi^{2}{ }_{0.05[1]}=3.84$; and $\chi^{2}=0.29<$ $\chi_{0.05[1]}^{2}=3.84$, respectively) (Fig. 2b).

The genetic stability of the GSS was monitored over several generations in mass rearing. No aberrant phenotypes were detected, i.e., under conditions the strain is stable. In addition, deviations from Hardy-Weinberg equilibrium were not detected using these polymorphic loci in the mass-reared colony. This genetic equilibrium suggests that our mass-rearing conditions had maintained genetic diversity, at least among these polymorphic loci tested for 12 generations.

\section{Mating competitiveness tests}

Eggs were collected from the infested egg balls in the field cage experiments. The average and standard error (SE) of the percentage of egg hatch for each treatment was used to estimate the level of the wild fertile female's sterility (Fig. 3). The egg hatch level from the baseline control treatment was relatively high but was not significantly different from a simulated MAT $(t=0.37$, d.f. $=4$, $P=0.730$ ).

Egg hatch rates of both the bisexual SIT and the maleonly SIT treatments were significantly lower than in the baseline control treatment, $(t=4.53$, d.f. $=4, P=0.011$ and $t=5.84$, d.f. $=4, P=0.004$, respectively). These results also showed that the male-only SIT practice was more effective than the traditional SIT using bisexual releases. The male-only SIT treatment generated more than 50 percent higher sterility rate when compared to the baseline control and MAT treatments (Fig. 3). This fact indicated that the mating competitiveness of the sterile Salayal males appeared to be equivalent to that of wild males.

The bisexual SIT in combination with MAT treatment generated significantly much higher level of sterility than the baseline control $(t=11.06$, d.f. $=4, P=0.000)$. Likewise, the male-only SIT combined with MAT treatment also generated significantly much higher level of sterility 


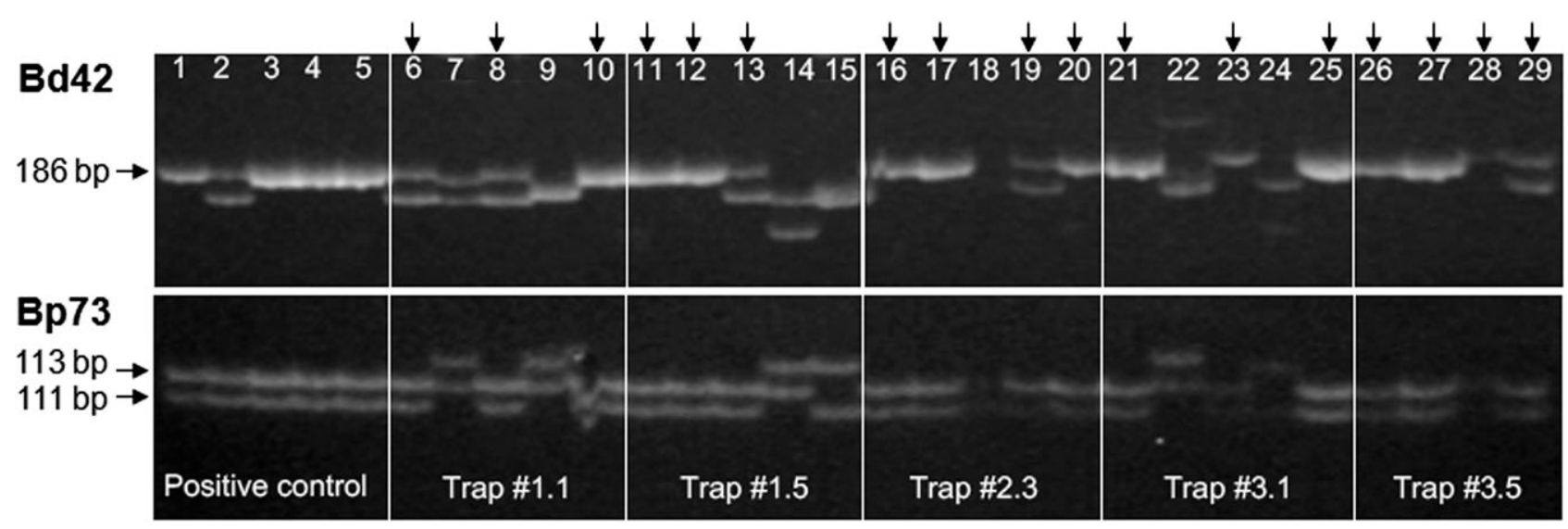

Fig. 4. Strain identification of the sterile released Salayal males from monitoring traps. Lanes 1 to 5 were positive control individuals. Arrows point at the expected genotypes of the sterile released males.

$(t=15.03$, d.f. $=4, P=0.000)$. This confirmed that the integrated pest management using SIT was very effective when MAT was integrated. In this case, the male-only SIT could be three times more effective than the application of bisexual SIT ( $t=2.69$, d.f. $=4, P=0.05$ ) (Fig. 3).

\section{Utilization of microsatellite markers for strain identification in traps}

Microsatellite loci Bd42 and Bp73 were used to identify Salayal males in weekly trapping samples involving Steiner traps (Fig. 4). The Bd42 and Bp73 loci were chosen because their $N_{\mathrm{a}}$ values in the wild population were high while the genotypic frequencies of the alleles $181 / 186$ or $186 / 186$ and $111 / 113$ were very low (Table 2 ). This would minimize the estimated possibility of false positives to less than about $0.2 \%$ or $[2 \times(0.01 \times 0.09)]$. Moreover, the genotyping analysis could be done on the same gel electrophoresis because the fragment size ranges of the two loci do not overlap. The quality of the DNA isolated from dead, trapped flies was sufficient for genotyping.

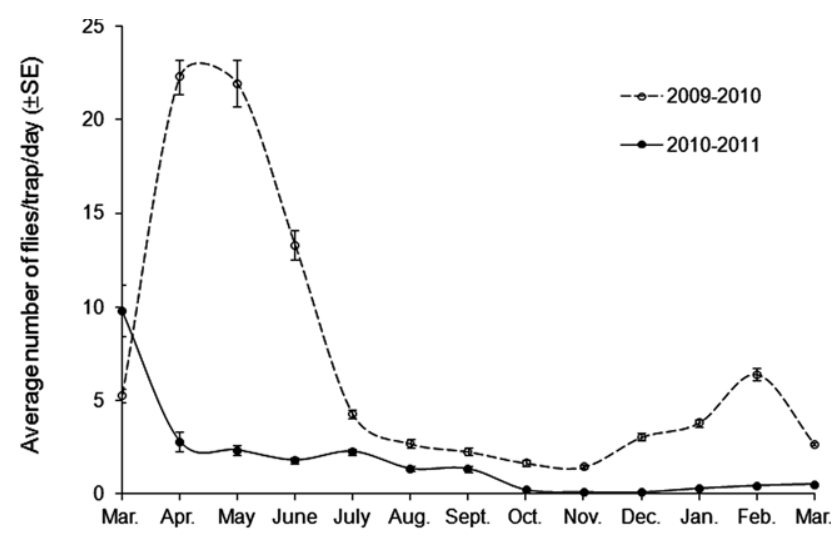

Fig. 5. Average number of wild oriental fruit flies (flies/trap/day $\pm \mathrm{SE}$ ) in tested fruit orchard; dashed line represents baseline data one year before the field trial; solid line represents data during the application of IPM using male-only SIT from the Salaya1 strain.

\section{Evaluation of the Salaya1 GSS in a pilot-scale IPM experiment using SIT}

The annual fruit fly prevalence before the utilization of the Salaya1 GSS for SIT was determined in a pilot fruit orchard (Fig. 5). The peak of the fruit fly abundance appeared to be synchronous to the mango season from March to June. The application of IPM using male-only SIT resulted in the suppression of the fruit fly population throughout the year. The fruit infestation rate was also reduced from approximately $30 \%$ to $5 \%$ after the SIT application.

\section{DISCUSSION}

The average genetic variability in the mass-reared Salayal strain was low. On the contrary, previously characterized mass-reared strain for bisexual releases showed a very high level of genetic variation $\left(N_{\mathrm{a}}=11.00, H_{\mathrm{O}}=\right.$ 0.71 , and $H_{\mathrm{E}}=0.86$ ) because such populations had been periodically refreshed with wild material from different geographical locations (Aketarawong et al., 2011). The small genetic variability of the Salayal strain was not unexpected. The genetic sexing strain (GSS) represented a special situation with respect to genetic diversity. The fact that such a strain was started with a single male and a very few individual white pupae mutants means that the descendants are expected to have reduced genetic diversity unless additional outcrosses had been performed. There was also the limitation that the overall genetic diversity can only be increased in the GSS by replacing all autosomes that were not involved in the sexing system and the $\mathrm{X}$ chromosome. This was because the autosomes involved in the sexing system were fixed and had to be maintained (Fig. 1). One of the most common concerns is that the lower genetic diversity of a strain may lead to a reduction in strain vigour and mating compatibility resulting in lower performance in the field. However, the field cage tests suggest that the Salayal strain remains sexually compatible with the wild population. This may be because the Salayal strain has undergone the selection processes that tend to accumulate harmoniously interacting genes in its population gene pool. Nonetheless, fur- 
ther studies are necessary to address the issue of adaptation related to mating behaviour.

The tested wild population showed a high level of genetic variability comparable with the previously characterized populations from various geographical origins (Aketarawong et al., 2007). In addition, the genetic diversity $\left(N_{\mathrm{a}}=11.36, H_{\mathrm{O}}=0.52\right.$, and $\left.H_{\mathrm{E}}=0.83\right)$ was especially similar to a previously characterized population located in the nearby $(<100 \mathrm{~km})$ Ratchaburi Province $\left(N_{\mathrm{a}}=10.14\right.$, $H_{\mathrm{O}}=0.53$, and $H_{\mathrm{E}}=0.86$; Aketarawong et al., 2011). This level of genetic diversity appears to be typical for natural $B$. dorsalis populations, although, more and larger populations need to be tested to confirm this.

The maintenance of genetic variability under the massrearing conditions, during the generations $F_{5}$ to $F_{17}$ implied that undesirable effects such as selection and inbreeding may be avoided using the current mass-rearing management system. However, those phenomena may be insidious. Routine genetic monitoring should be maintained in the mass-rearing facility. A significant rise in the heterozygosity of the $\mathrm{Bd} 9$ locus indicates that genetic diversity can also be increased under mass-rearing conditions. More extensive genetic analysis would be required to clarify this specific observation. Nonetheless, the genetic analysis of microsatellite loci, Bd9, Bd19, Bd85B, and Bp173, offers a new approach to study the maintenance of genetic variability in mass-reared strains. Higher level of genetic diversity, from any strain outcrossing, could also be verified by monitoring newly introduced alleles of these microsatellite loci.

The field cage mating tests showed that the release of sterile flies reduced fertility of wild females suggesting that the released males are sufficiently competitive. Furthermore, the release of sterile males only is clearly more effective than the release of both sexes. Even so, the best results were obtained when the male-only SIT was combined with a MAT treatment. This result is similar to earlier studies comparing male-only and bisexual SIT (McInnis et al., 1994; Rendón et al., 2004). Nevertheless, a larger field evaluation is required to support this finding. The MAT-only treatment showed no difference in sterility rates when compared to the baseline control treatment although MAT was known to be an effective control practice (Steiner et al., 1965). It is also known that MAT only has an effect on population size if $99 \%$ of the males have previously been eliminated. The arbitrary reduction of wild males may not be enough to reduce the overall mating with the wild females in the field cage. Fruit fly males are known to be polygamous.

Several of the analysed microsatellite markers may be used to distinguish the Salayal males from wild flies and can, therefore, replace the use of fluorescent powder for strain identification. Fluorescent-marking techniques pose a health risk and cause errors in identification (Hagler \& Jackson, 2001). However, it may be better to combine several of these markers to ensure the accuracy of the identification.

Several Y-pseudo-linked microsatellite markers were serendipitously characterized in the Salaya1 strain because of the Y-autosome translocation basis of the genetic sexing strain. Further cytogenetic study should be done to characterize the chromosomal translocation nature of this strain, as per the work of Zacharopoulou et al. (2011). Although the Y-pseudo-linked loci were applied for male-specific genotyping for practical strain identification, it is also conceivable that they could be developed and used in future sperm marking applications involving SIT. The effectiveness of SIT relies on mating performance between the released sterile males and wild females. Detection of molecular markers from the fruit fly sterile male sperm in the wild female spermathecae provides a direct method to assess performance of the sterile males in area-wide SIT programmes (San Andrés et al., 2007; Scolari et al., 2008).

The small-scale SIT application shows that the utility of the microsatellite markers for the management of the Salayal strain is practical. The application of the Salayal GSS for IPM-SIT suppressed the $B$. dorsalis populations and reduced the amount of infested fruit. An adequate level of fruit fly suppression during the mango season was not accomplished in the bisexual SIT trials described by Orankanok et al. (2007). In addition, the male-only SIT can be continued during the fruit growing season because there are no females to sting the ripened fruits. But a larger field evaluation should of course be performed as part of an area-wide integrated fruit fly management. This would also allow for an economic feasibility assessment to be done along with improvement of the quality management system for $B$. dorsalis male-only SIT.

\section{CONCLUSIONS}

The characterization and application of microsatellite markers in the Salayal GSS provides a new approach for $B$. dorsalis SIT management. Methods to evaluate the maintenance of genetic variability under mass-rearing conditions using microsatellite markers for strain identification are, as presently demonstrated, now available. In addition, the Y-pseudo-linked markers provide an opportunity to further develop sperm marking for the study of mating performance of sterile males. The mating competitiveness of the male fly was validated in field cages and a pilot-scale IPM programme. The strain is thus ready for use and evaluation in much larger area-wide control programmes.

ACKNOWLEDGEMENTS. The authors gratefully acknowledge two anonymous reviewers for their suggestions and R. Bachtell Eastland for his helpful editing comments on the manuscript. This research was supported by International Atomic Energy research contract no. 15600 as part of the Agency's Coordinated Research Project: Development and Evaluation of Improved Strains of Insect Pests for SIT to S. Thanaphum. A portion of this study is a partial requirement of the Master of Science degree thesis of S. Isasawin under the supervision of S. Thanaphum at the Department of Biotechnology, Faculty of Science, Mahidol University. 


\section{REFERENCES}

Aketarawong N., Bonizzoni M., Malacrida A.R., Gasperi G. \& Thanaphum S. 2006: Seventeen novel microsatellite markers from an enriched library of the pest species Bactrocera dorsalis sensu stricto. Mol. Ecol. Notes 6: 1138-1140.

Aketarawong N., Bonizzoni M., Thanaphum S., Gomuski L.M., Gasperi G., Malacrida A.R. \& Guglielmino C.R. 2007: Inferences on the population structure and colonization process of the invasive oriental fruit fly, Bactrocera dorsalis (Hendel). Mol. Ecol. 16: 3522-3532.

Aketarawong N., Chinviniskul S., Orankanok W., Guglielmino C.R., Franz G., Malacrida A.R. \& Thanaphum S. 2011: The utility of microsatellite DNA markers for the evaluation of area-wide integrated pest management using SIT for the fruit fly, Bactrocera dorsalis (Hendel), control programs in Thailand. Genetica 139: 129-140.

CABI/EPPO 1998: Distribution Maps of Quarantine Pests for Europe. CAB International, Wallingford, $768 \mathrm{pp}$.

Clarke A.R., Armstrong K.F., Carmichael A.E., Milne J.R., RoDERICK G.K. \& YeAtes D.K. 2005: Invasive phytophagous pests arising through a recent tropical evolutionary radiation: the Bactrocera dorsalis complex of tropical fruit flies. Annu. Rev. Entomol. 50: 293-319.

Dieringer D. \& SChlÖtTerer C. 2003: Microsatellite Analyser (MSA): a platform independent analysis tool for large microsatellite data sets. Mol. Ecol. Notes 3: 107-111.

FAO/IAEA/USDA 2003: FAO/IAEA/USDA Manual for Product Quality Control and Shipping Procedures for Sterile Massreared Tephritid Fruit Flies. Version 5.0 IAEA, Vienna.

FrANZ G. 2005: Genetic sexing strains in the Mediterranean fruit fly, an example for other species amenable to large-scale rearing for the sterile insect technique. In Dyck V.A., Hendrichs J. \& Robinson A.S. (eds): Sterile Insect Technique: Principles and Practice in Area-wide Integrated Pest Management. Springer, Dordrecht, pp. 427-451.

Goldstein D.B. \& Schlötterer C. 1999: Microsatellites: evolution and applications. www.amazon.com/MicrosatellitesEvolution-Applications-David-Goldstein/dp/0198504071.

Guichoux E., Lagache L., Wagner S., Chaumeil P., Léger P., Lepais O., Lepoittevin C., Malausa T., Revardel E., Salin F. \& Petit R.J. 2011: Current trends in microsatellite genotyping. Mol. Ecol. Res. 11: 591-611.

HAGLeR J.R. \& JACKSON C.G. 2001: Methods for marking insects: current techniques and future prospects. Annu. Rev. Entomol. 46: 511-543.

Hendrichs J., Kenmore P., Robinson A.S. \& Vreysen M.J.B. 2007: Area-wide integrated pest management (AW-IPM): principle, practice, and prospects. In Vreysen M.J.B., Robinson A.S. \& Hendrichs J. (eds): Area-wide Control of Insect Pests: From Research to Field Implementation. Springer, Dordrecht, pp. 3-33.

IAEA 2003: Trapping Guidelines for Area-wide Fruit Fly Programmes. Vienna, pp. 1-47.

KLASSEN W. 2005: Area-wide integrated pest management and the sterile insect technique. In Dyck V.A., Hendrichs J. \& Robinson A.S. (eds): Sterile Insect Technique: Principles and Practice in Area-wide Integrated Pest Management. Springer, Dordrecht, pp. 39-68.

Klassen W. \& Curtis C.F. 2005: History of the sterile insect technique. In Dyck V.A., Hendrichs J. \& Robinson A.S. (eds): Sterile Insect Technique: Principles and Practice in
Area-wide Integrated Pest Management. Springer, Dordrecht, pp. 3-36.

LoXDALE H.D. \& Lushai G. 1998: Molecular markers in entomology (Review). Bull. Entomol. Res. 88: 577-600.

McComBS S.D. \& SAUL S.H. 1995: Translocation-based genetic sexing system for the oriental fruit fly (Diptera: Tephritidae) based on pupal color dimorphism. Ann. Entomol. Soc. Am. 88: 694-698.

McInnis D.O., Tam S.Y., Grace C. \& Miyashita D. 1994: Population suppression and sterility rates induced by variable sexratio, sterile insect releases of Ceratitis capitata (Diptera: Tephritidae) in Hawaii. Ann. Entomol. Soc. Am. 87: 231-240.

Orankanok W., Chinvinijkul S., Thanaphum S., Sittilob P. \& ENKERLIN W.R. 2007: Area-wide integrated control of oriental fruit fly Bactrocera dorsalis and guava fruit fly Bactrocera correcta in Thailand. In Vreysen M.J.B., Robinson A.S. \& Hendrichs J. (eds): Area-wide Control of Insect Pests: From Research to Field Implementation. Springer, Dordrecht, pp. 517-526.

Rendón P., McInnis D., Lance D. \& Stewart J. 2004: Medfly (Diptera: Tephritidae) genetic sexing: large-scale field comparison of males-only and bisexual sterile fly releases in Guatemala. J. Econ. Entomol. 97: 1547-1553.

Rice W.R. 1989: Analysis tables of statistical tests. Evolution 43: $223-225$.

Robinson A. 2002: Genetic sexing strains in medfly, Ceratitis capitata sterile insect technique programmes. Genetica 116: $5-13$.

RousSET F. 2008: GENEPOP'007: a complete re-implementation of GENEPOP software for Windows and Linux. Mol. Ecol. Res. 8: 103-106.

San Andrés V., Urbaneja A., Sabater-Muñoz B. \& Castañera P. 2007: A novel molecular approach to assess mating success of sterile Caratitis capitata (Diptera: Tephritidae) males in sterile insect technique programs. J. Econ. Entomol. 100: 1444-1449.

Scolari F., Schetelig M.F., Bertin S., Malacrida A.R., GasPERI G. \& WIMMER E.A. 2008: Fluorescent sperm marking to improve the fight against the pest insect Ceratitis capitata (Wiedemann; Diptera: Tephritidae). New Biotechnol. 25: 76-84.

Shearman D.C.A., Gilchrist A.S., Crisafulli D., Graham G., LANGE C. \& Frommer M. 2006: Microsatellite markers for the pest fruit fly, Bactrocera papayae (Diptera: Tephritidae) and other Bactrocera species. Mol. Ecol. Notes 6: 4-7.

Steiner L.F., Mitchell W.C., Harris E.J., Kozuma T.T. \& FujIмото M.S. 1965: Oriental fruit fly eradication by male annihilation. J. Econ. Entomol. 58: 961-964.

Stephens A.E.A., Kriticos D.J. \& Leriche A. 2007: The current and future potential geographic distribution of the oriental fruit fly, Bactrocera dorsalis, (Diptera: Tephritidae). Bull. Entomol. Res. 97: 369-378.

Waits L.P., Luikart G. \& TaberLet P. 2001: Estimating the probability of identity among genotypes in natural populations: cautions and guidelines. Mol. Ecol. 10: 249-256.

WIMMER E.A. 2005: Eco-friendly insect management. Nat. Biotechnol. 23: 432-433.

Zacharopoulou A., Augustino A.A., SAyed W.A.A., Robinson A.S. \& Franz G. 2011: Mitotic and polytene chromosomes analysis of the oriental fruit fly, Bactrocera dorsalis (Hendel) (Diptera: Tephritidae). Genetica 139: 79-90.

Received December 6, 2011; revised and accepted March 15, 2012 